\title{
Human-induced pluripotent stem cells as a source of hepatocyte-like cells: new kids on the block
}

\author{
C. Busletta $\cdot$ E. Novo $\cdot$ M. Parola
}

Received: 28 March 2011 / Accepted: 30 June 2011/Published online: 23 July 2011

(C) Asian Pacific Association for the Study of the Liver 2011

One of the most exciting recent discoveries in the field of biology was the demonstration that both mouse and human somatic cells engineered via epigenetic reprogramming for the expression of combinations of few defined transcription factors (including Oct4, KLF4, Sox2, NANOG, LIN28, and c-Myc) can become pluripotent [1-3]. These cells closely resemble embryonic stem (ES) cells for their pluripotency and have been defined as induced pluripotent stem (iPS) cells. These cells have rapidly emerged as very promising tools for the achievement of significant advancement in different fields without the controversies and ethical concerns associated with the use of human ES cells [4]. In particular, the possibility to get iPS cells from readily obtainable somatic cells theoretically opened the way to a number of perspectives and practical applications, including (1) to design and test patient-customized (i.e., autologous) cell therapy without the need for immune suppression and (2) to increase our knowledge on mechanisms of inherited diseases with a realistic opportunity, as recently shown for human iPS-derived hepatocytes [5-7], for modeling inherited metabolic human diseases, understanding disease pathogenesis, and for drug discovery and testing. It should be anticipated, however, that the latter objective (i.e., the use of human iPS cell-derived hepatocytes for drug discovery and testing) can currently be envisaged as the most useful and safe application. Indeed, a number of relevant limitations have progressively emerged in relation to the clinical use of iPS cells and different

C. Busletta $\cdot$ E. Novo $\cdot$ M. Parola $(\bowtie)$

Department of Experimental Medicine and Oncology,

InterUniversity Center for Hepatic Pathophysiology,

University of Torino, Corso Raffaello 30, 10125 Turin, Italy

e-mail: maurizio.parola@unito.it laboratories have developed strategies in order to at least partially overcome such limitations.

The original methods used in order to derive iPS cells from either human or mouse somatic cells employed viral vectors, a strategy leading to the integration of both desired transgenes and vector backbone into the host cell genome [1-3]. Of relevance, the use of these vectors is at risk to produce insertional mutations and genetic alterations able to interfere with normal functions of cells derived from iPS cells and to favor reactivation of reprogramming factors at later stages. Indeed, residual transgene expression has been reported to affect differentiation into specific lineages [2] or even result in tumorigenesis [8-10]. At present, the oncogenic potential of iPS cells represents a major concern for clinical application [9] since these cells, much as ES cells, can readily form teratomas when injected into immunodeficient mice. This may rely, for both iPS and ES cells, on the presence of residual diploid pluripotent cells that have not undergone differentiation in the population of transplanted human ES or iPS cells [9]. Moreover, tumor formation in iPS cell chimeric mice has been attributed to the expression of c-Myc in iPS cell-derived somatic cells, irrespective of the type of the original somatic cell employed [9-13]. However, as well reviewed [9], several other reasons are likely to underlie iPS cell tumorigenicity. In particular, although iPS cells may behave similarly to ES cells, differences between these two kinds of cells indeed exist and this is particularly significant for human cells, with human iPS cells being more tumorigenic than human ES cells because of genetic and epigenetic causes [9].

Along these lines, human iPS cells have been reported to acquire chromosomal alterations and genetic changes (including aneuploidy, gain or loss of small chromosomes, and point mutations) even more readily than ES cells 
[9, 14]. Human ES cells, which are derived from the inner mass of blastocysts, may acquire chromosomal abnormalities mainly following prolonged growth in culture. By contrast, human iPS cells can acquire chromosomal alterations as a result of multiple events, including (1) longterm culture of somatic cells of origin, that are more likely to then carry genetic abnormalities which may be even selected during reprogramming; (2) the stress associated with reprogramming itself; and (3) long-term passaging of iPS cells in culture $[9,14]$.

In addition, increased tumorigenicity of human iPS cells versus ES cells can also depend on epigenetic differences. It has been recently proposed that global gene expression patterns between these two kinds of cells may differ significantly, although prolonged culture may bring iPS and ES cells transcriptionally closer [15-17]. In particular, human iPS cells have been reported to overexpress cancerrelated miRNAs as well as to carry alterations in cancerspecific gene promoter DNA methylation that arise during reprogramming to persist thereafter. Even more interesting, it has recently been proposed that both human and mouse iPS cells may retain "epigenetic memory" of their cells of origin [18, 19]. Such epigenetic memory may affect tumorigenicity either directly as a memory of cellular epigenetic transformation, or indirectly as a memory of epigenetic characteristics that make some kind of cells more vulnerable to transformation [9]. This memory has been described in a study that analyzed a large-scale methylation profile of several somatic donor cells; this study led to the identification of aberrant methylation in a very high number of sites, with only some of them being reversed after reprogramming in iPS cells [20]. One should also note that some human iPS cells have been reported to show aberrant silencing or activation of imprinted genes during the reprogramming process [21], whereas human ES cells usually exhibit fairly stable genomic imprinting at early stages $[22,23]$.

Where cell cycle and replicative potential are concerned, two more comments should be added. First, human iPS cells have been reported to acquire during reprogramming the distinct cell cycle properties of ES cells which allow them to divide independently on exogenous mitogenic signals (i.e., abbreviated gap phases, high proportion of cells in the $\mathrm{S}$ phase and in the $\mathrm{M}$ phase of the cycle). However, as recently noted [9], the risk for reactivation of the reprogramming factors (particularly c-Myc) and the mentioned risk for acquiring genetic abnormalities during propagation of iPS in culture are likely to render human iPS cells more vulnerable to cell cycle aberrations. Second, the use of potent oncogenes for somatic cell reprogramming as well as the involvement of p53 pathway as a defensive barrier against this process and the mentioned epigenetic memory may raise the risk for human iPS cells to lead even to the development of somatic tumors (i.e., something which is unlikely to apply for ES cells).

Thus, the first general take-home message is that we are still far from the goal to generate safe human iPS-derived cells for clinical applications. This goal, according to what was recently proposed, should require terminal differentiation or accurate elimination of residual pluripotent stem cells or, alternatively, the effective ability to interfere with tumor progression genes (i.e., to prevent tumor formation from residual pluripotent cells) [9].

Nonetheless, several alternative approaches have been developed in the recent years that have started to overcome some of the problems and limitations of the original protocols for iPS induction. Along these lines, the Thomson research group [2] made a relevant contribution by creating human iPS cells from postnatal adult foreskin fibroblasts adopting a different approach. These authors, by employing a lentiviral system to test candidate stemness-related genes, showed that reprogramming somatic cells for the expression of Oct4, Sox2, Nanog, and Lin28 genes was a strategy sufficient to obtain human pluripotent stem cells [6]. These cells retained relevant characteristics of human ES cells avoiding the oncogenic potential related to c-Myc reprogramming [6, 8-10]. More recently, it has been shown that reprogramming of Oct4, Sox2, and Klf4 expression was sufficient to obtain iPS cells from both mouse and human somatic cells [24]. Although these c-Myc-lacking iPS cells were characterized by a much lower efficiency (i.e., if compared to iPS cells obtained by reprogramming $\mathrm{c}-\mathrm{Myc})$, mice derived from these cells did not develop tumors during the study period [24].

Since a major challenge in the field was to design methods involving minimal modification to the genome in order to generate iPS cells to be used safely for cell therapy [25], several laboratories introduced other alternative procedures. Two different studies have reported protocols for the derivation of iPS cells in which the procedure leading to integration of the four reprogramming factors, based on the use of plasmids [26] or lentiviruses [27], was followed by removing transgene sequences from the host genome by means of Cre/lox-mediated excision. Although this procedure resulted in the successful removal of transgene sequences, it apparently (see also comments in [28]) left behind residual vector sequences. Another approach was described in the study by Woltjen et al. [29] where piggyBac transposons (a system requiring only the inverted terminal repeats flanking a transgene and transient expression of the transposase enzyme to catalyze insertion or excision events) were employed to obtain vector-free murine iPS cells. This approach, although elegant and potentially interesting, has been questioned for two main reasons: (1) the lack of a definitive proof for effective removal of vector from these cells and (2) the hard and 
difficult work needed to remove multiple transposons. Along these lines, another strategy was employed by the group of Thomson [28]; these authors obtained vector- and transgene-free iPS cells from human fibroblasts following a procedure requiring a single transfection with oriP/ Epstein-Barr nuclear antigen-1 (EBNA1)-based episomal vectors. These episomal vectors did not integrate in the genome and were completely removed from iPS cells, which being free from vectors and transgenes were reported to behave (proliferation and developmental potential) similarly to human ES cells.

A further recent development in iPS cell generation has been the so-called "protein transduction" approach, a possible alternative to the nucleic-acid-based one for the reprogramming of somatic cells into iPS cells without genetic manipulation [30]. The generation of stable iPS cells from mouse fibroblasts has been reported by protein transduction of recombinant reprogramming factors Oct4, Sox2, Klf4, and c-Myc (designed as poly-arginine protein transduction domain fused to the C-terminus of the reprogramming factors). These factors were found to enter target cells, remain stable within the cells for $\geq 48 \mathrm{~h}$, and translocate to the nucleus. Interestingly, the design of the protocol required four cycles of exposure of mouse fibroblasts to recombinant factors in a medium supplemented with valproic acid (VPA), a histone deacetylase (HDAC) inhibitor that can significantly improve reprogramming efficiency [30]. This helps to introduce a related approach, still designed to avoid or minimize genetic manipulation, in which small molecules are currently investigated and proposed as effective tools to modulate ES-cell fate and reprogramming of somatic cells. Since the field is a rapidly expanding one (for a comprehensive review see [31]) here is sufficient to say that different laboratories have provided evidence that several small molecules, including HDAC inhibitors (trichostatin A, suberoylanilide hydroxyamic acid, VPA, and other drugs under development) and DNAmethyltransferase inhibitors (5-azacytidine and RG108) can improve mouse and human somatic cell reprogramming $[31,32]$. Some of these molecules improved derivation of iPS cells from human fibroblasts that were reprogrammed for the expression of only two factors (Oct4 and Sox2) [33]. To the list of small molecules able to promote somatic reprogramming, one may add in the future a number of agents able to specifically affect signal transduction pathways (by targeting MEK, GSK3, PKC, etc.) that at present have been employed mostly on ES cells (reviewed in [31]).

The discovery of iPS cells has made them an attractive cellular source, ethically acceptable and potentially unlimited, of human hepatocyte-like cells for the study and treatment of human liver diseases. At present, there is an urgent need for generation of iPS cells, as a therapeutic approach particularly for end-stage chronic liver diseases
(CLDs) that represent a worldwide significant cause of morbidity and mortality. Moreover, although orthotopic liver transplantation (OLT) can be considered as a reliable and efficient therapeutic approach for acute liver failure (ALF), CLDs, and non-metastatic liver cancer, the shortage of organ donors and morbidity related to long-term immune-suppression therapy represent real concerns. The use of primary/adult hepatocyte theoretically should represent the best choice for clinical applications, and indeed some positive results have been reported in the past such as successful transplantation of these cells in human patients suffering from either ALF or selected metabolic diseases [34-37]. However, the use of primary/adult hepatocytes for clinical applications recognizes a number of limitations: (1) any strategy involving direct cell therapy (i.e., transplantation of adult hepatocytes) needs the availability of a very high number of cells, which is very difficult to achieve; (2) cultured hepatocytes are characterized by a slow growth rate and, most relevant, usually undergo significant loss of function and de-differentiation; and (3) similar limitations have been described for the use of hepatocytes in order to investigate drug toxicity or for human disease modeling [34-38]. From an historical point of view, these limitations first led several laboratories to explore different cellular sources for their potential to differentiate in vitro into hepatocyte-like cells or to be transplanted to achieve liver engraftment and hepatocyte-like differentiation in vivo. Cellular sources such as embryonic or fetal hepatocytes, hepatic stem/progenitor cells, and extrahepatic stem cells (mostly bone marrow-derived) have been extensively used in the last decade and results from these studies have been recently critically reviewed and their major limitations (ethical, technical, etc.) emphasized [36, 37, 39-41]. In particular, the use of human ES cells faces significant ethical concerns in several countries, although these cells have been shown to efficiently differentiate into hepatocytes [42]. Having in mind this scenario, different laboratories started to work on iPS cells and, as recently reviewed and discussed [7, 25, 42, 43], succeeded in differentiating hepatocyte-like cells from either murine $[44,45]$ or human $[5,6,46,47]$ iPS cells.

Where human iPS cells are concerned, differentiation into hepatocyte-like cells has been achieved using protocols derived from those originally designed for human ES cells [48-50]; these protocols may differ in some details but have a relatively common unifying scheme which involves 3-4 steps in order to obtain hepatocyte-like cells. The protocol adopted in a study from the group of Duncan [40] may serve as a general reference. These authors employed human normal skin fibroblasts in which iPS induction was obtained by engineered expression of Oct4, Sox2, Nanog, and Lin28. iPS cells were then submitted to a four step protocol: 
1. iPS cells were first exposed to activin A $(100 \mathrm{ng} / \mathrm{ml})$ in RPMI medium containing also B27 supplements for 5 days, a procedure which efficiently induces differentiation of definitive endoderm, as already shown for hESC [51, 52].

2. Hepatic specification was then obtained by exposing the cells for additional 5 days to RPMI/B27 medium containing bone morphogenetic protein-4 (BMP-4, $20 \mathrm{ng} / \mathrm{ml}$ ) and fibroblast growth factor-2 (FGF-2, $10 \mathrm{ng} / \mathrm{ml}$ ) at a lower $\mathrm{pO}_{2}(4 \%)$, which is known to contribute to maintain, through interrelated action of hypoxia-inducible factor (HIF)-1 and -2, properties of stem cells [53].

3. Proliferation of immature hepatocyte-like cells was achieved by exposing cells to RPMI/B27 medium containing hepatocyte growth factor (HGF, $20 \mathrm{ng} / \mathrm{ml}$ ) still at $4 \% \mathrm{pO}_{2}$.

4. Maturation to hepatocyte-like cells was finally obtained at a $20 \% \mathrm{pO}_{2}$ by adding hepatocyte culture medium (HCM) containing oncostatin M (OSM, $20 \mathrm{ng} / \mathrm{ml}$ ) for additional 5 days.

At the end of this protocol the reported average number of albumin-positive human iPS-derived hepatocytes was $81.0 \pm 4.8 \%$ [30] and these cells were reported to have a convincing morphology (including some bi-nucleated cells) and, most important, to express convincing features of hepatocyte differentiation (see following text).

Although such a general protocol to obtain human iPSderived hepatocyte-like cells may be in principle satisfying and quite simple to use, one may observe that it is potentially expensive since it requires the use of several cytokines and, even more relevant, is time-consuming. To obtain immature and mature hepatocyte-like cells, the protocol requires $\geq 15$ and 20 days, respectively. As far as clinical application such as autologous cell therapy may be envisaged, human patients (i.e., particularly those experiencing ALF) may have indeed a limited chance to survive such a period of time.

In the current issue of the journal, Takata and coworkers [54], in a study designed to address the previous considerations, describe what is likely to be seen as a promising improvement of the original general protocol to obtain hepatocyte like-cells from human iPS cells. Although these authors referred to the same general scheme derived from $\mathrm{hESC}$ protocols, they succeeded in a consistent shortening of the procedure, reducing the use of recombinant cytokines to a minimum. The Takata et al. [54] protocol, in particular, still requires the use of a shortened step ( 3 days instead of 5) to obtain definitive endoderm cells by exposing them to RPMI/B27 medium containing activin A $(100 \mathrm{ng} / \mathrm{ml})$, but significantly omits the 5-days hepatic specification step based on the exposure to medium containing BMP-4 and FGF-2. This protocol allowed to obtain immature and mature (i.e., after OSM-related maturation step) hepatocyte-like cells within 8 and 13 days, respectively, instead of 15 and 20 as for the original protocol. Moreover, data provided by authors apparently indicate that the shortened and simplified procedure was sufficient to obtain a scenario of definitive endodermal cells (as deduced by evaluation of FoxA2 and Sox17) and then of hepatocyte-like differentiation. Hepatocyte-like phenotype was ascertained by testing albumin secretion, urea formation, glycogen storage, cytochrome P450 3A4 (CYP3A4) activity, and hepatocyte nuclear factor $4 \alpha$ (HNF4 $\alpha$ ) transcription, and found as efficient as the original and longer protocol or even superior for certain features.

Although data from Takata et al. [54] study are in principle of interest, a number of cautionary notes should be taken into account. As a first comment, the fact that the cells were showing to express some appropriate stagespecific markers and hepatocyte-like functions does not allow to definitively establish how the cells at the end of the protocol were really similar to mature primary human hepatocytes. Indeed, any study designed to generate hepatocyte-like cells should always offer a wide analysis of the acquisition of enzymatic, biochemical, and other protein functions typical of mature/adult hepatocytes. This should include other essential features in addition to those mentioned, including (see e.g., $[5,6]$ ) the capacity to accumulate lipids and to actively take-up low-density lipoproteins. An analysis of the activity of different specific cytochrome P450 isoforms and other enzymes involved in drug disposal as well as of the effective ability to metabolize agents or drugs (i.e., to more efficiently test phase I and phase II drug metabolizing efficiency) should be provided. This can be accompanied by an analysis of liverspecific mRNA transcription after differentiation and compared to undifferentiated iPS cells, as in the Duncan's group study [40], but transcriptome analysis should be envisaged just as an additional approach to the phenotypic/ functional one.

Still concerning the efficiency of the protocol, an additional comment should be raised that is substantially similar to the one addressed by others [7] when commenting a seminal study addressing the use of human iPS-derived hepatocytes for modeling inherited metabolic human diseases in cell culture systems [5]. Data from Takata et al. [54] study indicate that at the end of their protocol a significant percentage of putative hepatocyte-like cells were still $\alpha$-fetoprotein (AFP) positive, making then uncertain to understand how well the protocol-related differentiation process was recapitulating the normal development and function of hepatocytes. Along these lines, it should be also of interest to know whether during the in vitro 
differentiation process iPS cells express markers of hepatic progenitor cells (e.g., cytokeratin 7 or 19, HepCAM).

On the contrary, it is interesting to note that Takata et al. [54] did not find relevant differences in hepatocyte-like differentiation when using two different parental iPS cell populations. This is a rather critical point that should be further addressed and validated in the future by running in parallel this kind of experiments (i.e., differentiation of hepatocyte-like cells) on a more significant number of parental iPS cells. This is imperative since it has been reported in the literature that variability in response to differentiation toward neuronal or hemopoietic lineages among iPS lines, either from the same source or from different sources, may exist [55, 56].

A final, but in our view, substantial series of critical issues should be considered. In an ideal study, the characterization of hepatocyte-like functions should be always followed by specific and carefully designed experimental approaches in order to offer a more definitive validation of the reliability of differentiated hepatocyte-like cells according to the specific purpose of the study. As an example, if cell therapy is the goal, the study should provide specific in vivo evidence that iPS-derived hepatocytelike cells may engraft the liver (see [6] where authors checked this by injecting differentiated human cells into the right lateral liver lobe of newborn mice and then carefully checked engraftment of these cells) and/or display the ability to safely repopulate damaged liver. The study by Takata et al. [54] unfortunately does not provide such in vivo evidence and one cannot avoid to note that these authors have reprogrammed human somatic cells using lentiviruses in order to transduce a combination of factors which still include c-Myc (at least in some cells). Moreover, the authors applied none of those previously mentioned procedures which have been developed in order to eventually obtain vector- and transgene-free cells. On the basis of what we discussed before (tumorigenicity and other concerns), these are likely to represent substantial limitations for a cell therapy application. Indeed, here we come once again to a more general critical consideration: at present the use of human iPs-derived hepatocyte-like cells for cell therapy and regenerative medicine is still far from being a reliable and safe application whereas, based on literature data, the most useful applications for human iPSderived cells are for disease modeling, drug discovery and testing, and toxicological purposes.

At present, at least to our knowledge, just a single murine study has provided evidence that somatic cells from wild-type mice reprogrammed to iPS cells may have the potential to undergo in vivo normal ontogenic development into mature hepatocytes in the experimental model of mice carrying fumarylacetoacetate hydrolase deficiency (FAH -/- mice) [57]. In that study, wild-type murine iPS cells were injected into blastocysts of FAH $-/-$ mice giving rise to a generation of chimeric mice in which iPS-derived hepatocytes were able to proliferate (even responding to two/third partial hepatectomy) and repopulate the liver rescuing the chimeric mice. However, two major comments should be raised on this otherwise elegant study: (1) the FAH $-/-$ mice is a rather unique and favorable model in the scenario of regenerative medicine applied to hepatology; indeed, most of the studies published in the last decade and referring to either acute or chronic conditions of experimental liver injury have reported that engraftment and proliferation of hepatocyte-like cells from human ESC and iPS cells (as for most studies employing bone marrowderived stem cells) in rodents after transplantation are quite limited and then mostly disappointing [reviewed in [37, 39-42]) and (2) even more important, a model in which transfer of iPS cells are injected into blastocysts should be considered as mostly irrelevant for regenerative medicine where the final goal is to obtain safe and adult/mature hepatocyte-like cells to transplant under postnatal settings.

In our view, an excellent example of a realistic study on the actual possible application of human iPS-derived hepatocyte-like cells is the one recently published by Rashid et al. [5] which has investigated the potential of these cells to model inborn liver diseases. These authors obtained human dermal fibroblasts from patients affected by a number of inherited metabolic disorders, including $\alpha 1$-antitrypsin deficiency (AAT), glycogen storage disease type 1a deficiency (GSD1), hereditary tyrosinemia type 1, Crigler-Najjar syndrome, and familial hypercholesterolemia (FH). They first generated a library of patient-specific human iPS cells to be then differentiated into hepatocytelike cells and then characterized iPS cell-derived hepatocytes from patients affected by AAT, GSD1, and FH. As noted by Greenbaum [43], AAT, GSD1, and FH are conditions in which three different mechanisms of liver disease are involved: (1) defective protein secretion, (2) deficiency of a intracellular enzyme, and (3) lack of a plasma membrane receptor, respectively. This study is relevant because iPS cell-derived hepatocytes were reported to exhibit all characteristic phenotypic abnormalities found in primary hepatocytes obtained from patients affected by these diseases. Moreover, although some limitations still exist, as acknowledged by authors and discussed in detail elsewhere [7, 43], such a kind of study can disclose the unique and exciting opportunity to test drugs that may be tailored to the single individual.

As a concluding comment, the study by Takata et al. [54] can be envisaged as one more positive technical step designed to accelerate the procedure to obtain iPS cellderived hepatocytes. However, one should be aware that more experimental work is needed to overcome current limitations as well as experimental and clinical challenges 
related to the use of these cells and that applications other than cell therapy are at present more realistic.

\section{References}

1. Takahashi K, Yamanaka S. Induction of pluripotent stem cells from mouse embryonic and adult fibroblast cultures by defined factors. Cell 2006;126:663-676

2. Yu J, Vodyanik MA, Smuga-Otto K, et al. Induced pluripotent stem cell lines derived from human somatic cells. Science 2007;318:1917-1920

3. Takahashi K, Tanabe K, Ohnuki M, et al. Induction of pluripotent stem cells from adult human fibroblasts by defined factors. Cell 2007;131:861-872

4. Yamanaka S, Blau HM. Nuclear reprogramming to a pluripotent state by three approaches. Nature 2010;465:704-712

5. Rashid ST, Corbineau S, Hannan N, et al. Modeling metabolic inherited disorders of the liver using human pluripotent stem cells. J Clin Invest 2010;120:3127-3136

6. Si-Tayeb K, Noto FK, Nagaoka M, et al. Highly efficient generation of human hepatocyte-like cells from induced pluripotent stem cells. Hepatology 2010;51:297-305

7. Soto-Gutierrez A, Tafaleng E, Kelly V, Roy-Chowdhury J, Fox IJ. Modeling and therapy of human liver diseases using pluripotent stem cells: how far we come? Hepatology 2011;53:708-711

8. Okita K, Ichisaka T, Yamanaka S. Generation of germlinecompetent induced pluripotent stem cells. Nature 2007;448: 313-317

9. Ben-David, Benvenisty N. The tumorigenicity of human embryonic and induced pluripotent stem cells. Nat Rev Cancer 2011;11:268-277

10. Yu J, Thomson JA. Pluripotent stem cell lines. Genes Dev 2008;22:1987-1997

11. Maherali N, Sridharan R, Xie W, et al. Directly reprogrammed fibroblasts show global epigenetic remodeling and widespread tissue contribution. Cell Stem Cell 2007;1:55-70

12. Wernig M, Meissner A, Foreman R, et al. In vitro reprogramming of fibroblasts into a pluripotent ES-cell like state. Nature 2007;448:318-324. v

13. Takashi A, Kojiro Y, Masato N, et al. Generation of pluripotent stem cells from adult mouse liver and stomach cells. Science 2008;321:699-702

14. Ben-David U, Benvenisty N, Mayshar Y. Genetic instability in human induced pluripotent stem cells: classification of causes and possible safeguards. Cell Cycle 2010;9:4603-4604

15. Chin MH, Mason MJ, Xie W, et al. Induced pluripotent stem cells and embryonic stem cells are distinguished by gene expression signatures. Cell Stem Cell 2009;5:111-123

16. Guenther MG, Frampton GM, Soldner F, et al. Chromatin structure and gene expression programs of human embryonic and induced pluripotent stem cells. Cell Stem Cell 2010;7:249-257

17. Newman AM, Cooper JB. Lab-specific gene expression signatures in pluripotent stem cells. Cell Stem Cell 2010;7:258-262

18. Polo JM, Liu S, Figueroa ME, et al. Cell type of origin influences the molecular and functional properties of mouse induced pluripotent stem cells. Nat Biotech 2010;28:848-855

19. Kim K, Doi A, Wen B, et al. Epigenetic memory in induced pluripotent stem cells. Nature 2010;467:285-290

20. Ron-Bigger S, Bar-Nur O, Isaac S, Bocker M, Lyko F, Eden A. Aberrant epigenetic silencing of tumor suppressor genes is reversed by direct reprogramming. Stem Cells 2010;28: 1349-1354
21. Pick M, Stelzer Y, Bar-Nur O, Mayshar Y, Eden A, Benvenisty N. Clone- and gene-specific aberrations of parental imprinting in human induced pluripotent stem cells. Stem Cells 2009;27: 2686-2690

22. Rugg-Gunn PJ, Ferguson-Smith AC, Pedersen RA. Status of genomic imprinting in human embryonic stem cells as revealed by a large cohort of independently derived and maintained lines. Hum Mol Genet 2007;16:R243-51

23. Adewumi O, Aflatoonian B, Ahrlund-Richter L, et al. Characterization of human embryonic stem cell lines by the International Stem Cell Initiative. Nat Biotech 2007;25:803-816

24. Nakagawa M, Koyanagi M, Tanabe K, et al. Generation of induced pluripotent stem cells without Myc from mouse and human fibroblasts. Nat Biotechnol 2008;26:101-106

25. Asgari S, Pournasr B, Salekdeh GH, Ghodsizadeh A, Ott M, Baharvand $\mathrm{H}$. Induced pluripotent stem cells: a new era for hepatology. J Hepatol 2010;53:738-751

26. Kaji K, Norrby K, Paca A, Mileikovsky M, Mohseni P, Woltjen $\mathrm{K}$. Virus-free induction of pluripotency and subsequent excision of reprogramming factors. Nature 2009;458:771-775

27. Soldner F, Hockemeyer D, Beard C, et al. Parkinson's disease patient-derived induced pluripotent stem cells free of viral reprogramming factors. Cell 2009;136:964-977

28. Yu J, Hu K, Smuga-Otto K, et al. Human induced pluripotent stem cells free of vector and transgene sequence. Science 2009;324:797-801

29. Woltjen K, Michael IP, Mohseni P, et al. piggyBac transposition reprograms fibroblasts to induced pluripotent stem cells. Nature 2009;458:766-770

30. Zhou H, Wu S, Joo JY, et al. Generation of induced pluripotent stem cells using recombinant proteins. Cell Stem Cell 2009;4: 381-384

31. Li W, Ding S. Small molecules that modulate embryonic stem cell fate and somatic cell reprogramming. Trends Pharmacol Sci 2010;31:36-45

32. Huangfu D, Maehr R, Guo W, et al. Induction of pluripotent stem cells by defined factors is greatly improved by small-molecule compounds. Nat Biotechnol 2008;26:795-797

33. Huangfu D, Osafune K, Maehr R, et al. Induction of pluripotent stem cells from primary human fibroblasts with only Oct4 and Sox2. Nat Biotechnol 2008;26:1269-1275

34. Fisher RA, Strom SC. Human hepatocyte transplantation: worldwide results. Transplantation 2006;82:441-449

35. Fitzpatrick E, Mitry RR, Dhawan A. Human hepatocyte transplantation: state of the art. J Intern Med 2009;266:339-357

36. Soto-Gutierrez A, Navarro-Alvarez N, Yagi H, Yarmush ML. Stem cells for liver repopulation. Curr Opin Organ Transplant 2009;14:667-673

37. Zern MA. Cell transplantation to replace whole liver transplantation. Gastroenterology 2009;136:767-769

38. Enns GM, Millan MT. Cell-based therapies for metabolic liver disease. Mol Genet Metab 2008;95:3-10

39. Alison MR, Islam S, Lim S. Stem cells in liver regeneration, fibrosis and cancer: the good, the bad and the ugly. J Pathol 2009;217:282-298

40. Duncan AW, Dorrell C, Grompe M. Stem cells and liver regeneration. Gastroenterology 2009;137:466-481

41. Russo FP, Parola M. Stem and progenitor cells in liver regeneration and repair. Cytotherapy 2011;13:135-144

42. Behbahan IS, Duan Y, Lam A, et al. New approaches in the differentiation of human embryonic stem cells and induced pluripotent stem cells toward hepatocytes. Stem Cell Rev 2011. doi: 10.1007/s12015-010-9216-4 (in press)

43. Greenbaum L. From skin cells to hepatocytes: advances in application of iPS cell technology. J Clin Invest 2010;120: 3102-3105 
44. Gai H, Nguyen DM, Moon YJ, et al. Generation of murine hepatic lineage cells from induced pluripotent stem cells. Differentiation 2010;79:171-181

45. Li W, Wang D, Qin J, et al. Generation of functional hepatocytes from mouse induced pluripotent stem cells. J Cell Physiol 2010; 222:492-501

46. Song Z, Cai J, Liu Y, et al. Efficient generation of hepatocyte-like cells from human induced pluripotent stem cells. Cell Res 2009; 19:1233-1242

47. Sullivan GJ, Hay DC, Park IH, et al. Generation of functional human hepatic endoderm from human induced pluripotent stem cells. Hepatology 2010;51:329-335

48. Agarwal S, Holton KL, Lanza R. Efficient differentiation of functional hepatocytes from human embryonic stem cells. Stem Cells 2008;26:1117-1127

49. Shiraki N, Umeda K, Sakashita N, Takeya M, Kume K, Kume S. Differentiation of mouse and human embryonic stem cells into hepatic lineages. Genes Cells 2008;13:731-746

50. Hay DC, Fletcher J, Payne C, et al. Highly efficient differentiation of hESCs to functional hepatic endoderm requires ActivinA and Wnt3a signaling. Proc Natl Acad Sci USA 2008;105:1230112306

51. D'Amour KA, Agulnick AD, Eliazer S, Kelly OG, Kroon E, Baetge EE. Efficient differentiation of human embryonic stem cells to definitive endoderm. Nat Biotechnol 2005;23:1534-1541
52. McLean AB, D'Amour KA, Jones KL, et al. Activin a efficiently specifies definitive endoderm from human embryonic stem cells only when phosphatidylinositol 3-kinase signaling is suppressed. Stem Cells 2007;25:29-38

53. Simon MC, Keith B. The role of oxygen availability in embryonic development and stem cell function. Nat Rev Mol Cell Biol 2008;9:285-296

54. Takata A, Otsuka M, Kogiso T, et al. Direct differentiation of hepatic cells from human-induced pluripotent stem cells using a limited number of cytokines. Hepatol Int 2011. doi:10.1007/ s12072-011-9251-5

55. Hu BY, Weick JP, Yu J, et al. Neural differentiation of human induced pluripotent stem cells follows developmental principles but with variable potency. Proc Natl Acad Sci USA 2010;107: $4335-4340$

56. Kulkeaw K, Horio Y, Mizuochi C, Ogawa M, Sugiyama D. Variation in hematopoietic potential of induced pluripotent stem cell lines. Stem Cell Rev 2010;6:381-389

57. Espejel S, Roll GR, McLaughlin KJ, et al. Induced pluripotent stem cell-derived hepatocytes have the functional and proliferative capabilities needed for liver regeneration in mice. J Clin Invest 2010;120:3120-3126 